\title{
Elongational viscosity by fiber spinning
}

\author{
Z. Chen and T.C. Papanastasiou ${ }^{1}$ \\ Macromolecular Research Center and ${ }^{1}$ Department of Chemical Engineering, University of Michigan, Ann Arbor, \\ Michigan, USA
}

\begin{abstract}
Isothermal melt, fiber-spinning was recently analyzed by means of a nonlinear, integral, constitutive equation that incorporates shear history effects, spectrum of relaxation times, shear-thinning, and extension thinning or thickening when either the drawing force or the draw ratio is specified. The predictions agreed with experimental data on spinning of polystyrene, low-density polyethylene, and polypropylene melts. The predicted apparent elongational viscosity along the threadline (which, as shown in this work, must be identical to that measured experimentally by fiber spinning type of elongational rheometers) is compared with the true elongational viscosity predicted by the same constitutive equation under well-defined experimental conditions of constant extension rate, independent of any strain history. It is concluded that the apparent elongational viscosity, as measured by fiber-spinning, approaches the true elongational viscosity at low Weissenberg numbers (defined as the product of the liquid's relaxation time multiplied by the extension rate). At moderate Weissenberg numbers, the two viscosities may differ by an order of magnitude and their difference grows even larger at high Weissenberg numbers.
\end{abstract}

Key words: Fiber-spinning; elongational viscosity; integral model

\section{Introduction}

The fiber-spinning process is an important industrial operation for synthetic fibers, where a viscoelastic liquid is extruded continuously through a die and is taken up downstream at a higher speed to the desired diameter. In addition to its application in industry, the fiber-spinning technique is used as an elongational rheometer with the advantage of generating high extension rates within liquids of low to high zero-shear viscosity $[1-7]$.

Besides, shear flow, elongational flow is another type of flow involved in many industrial processes, such as fiber spinning, film and sheet casting, curtain coating, film blowing, and others. It is also of interest from the molecular-theory point of view, because elongational flow is fundamentally different from shear flow. At the same time, the measurement of pure elongational response is relatively undeveloped compared to the shear response, because the elongational, unbounded flow is difficult to produce experimentally. In contrast to the behavior in shear flow, the behavior of many viscoelastic liquids in elongational flow is unclear, although there have been increasing numbers of studies on the elongational properties of viscoelastic liquids, based on fiber-spinning or otherwise [8-12] in recent years.

Isothermal fiber-spinning, in relation to the elongational viscosity of viscoelastic liquids, has received a great deal of attention both experimentally and theoretically. Chen et al. [13] studied melt fiber-spinning of low- and high-density polyethylene, and predicted that the apparent elongational viscosity of LDPE increases with extension rate, whereas that of HDPE decreases. Petrie [14] studied the same process theoretically, and concluded that the elongational viscosity measured in fiber spinning is not a unique function of the material and the extension rate, but also depends on the strain history. Larson [15] analyzed isothermal fiber spinning with the Doi-Edwards constitutive equation, which predicted that the elongational viscosity decreases with extension rate. Recently, Sridhar and Gupta [16] studied by fiberspinning the apparent elongational viscosity of polymeric solutions with the upper convected Maxwell model and the upper convected Jeffrey model, 
and found that the liquid exhibits a Newtonian behavior with an apparent elongational viscosity equal to three times its zero-shear viscosity and that the apparent elongational viscosity gradually increases from the Newtonian value as the extension rate increases. Papanastasiou et al. [17] analysed the same process by means of a nonlinear, integral, constitutive equation [18], and their predictions agreed with experimental data of polystyrene [2] and lowdensity-polyethylene [19] spinning. Following them, Chen and Papanastasiou [20] modeled the same process with the Curtiss-Bird [21] (and Doi-Edwards [22]) constitutive equation and predicted accurately the velocity profile and the drawing force (or the draw ratio) for polystyrene [2] and polypropylene [23]. Both integral constitutive equations used are known to model both extension-thinning and extensionthickening liquids.

In this study, the apparent elongational viscosity along the spinline is calculated by means of the same integral constitutive equations. This viscosity must be identical to that measured experimentally from the local-fiber profile and the tension on the spinneret, if the numerical modeling is to predict experimentally measured variables - the velocity (and the fiber) profile and the draw ratio or the drawing force - accurately. In fact, the elongational viscosity is fully determined from the fiber profile and the drawing force.

The true elongational viscosity is calculated by means of the same constitutive equations under welldefined conditions of constant-extension rate, independent of any strain history. These conditions, presently impossible to produce experimentally for liquids of not so high shear-viscosity, are those achieved in stretching highly viscous melts [24] or in lubricated flows under perfect slip $[11,25]$. The underlying idea of this work appears to be similar to the recent work by MacKay and Petrie [26], which was developed independently, on apparent elongational viscosity using the fiber-spinning and pure methods.

\section{True elongational viscosity}

The true elongational viscosity $\eta_{e}$, defined as the ratio of force to the area of strain rate, takes the form of the ratio of the first normal stress difference $\tau_{11}-\tau_{22}$ to a constant extension rate $\dot{\varepsilon}$ for an incompressible liquid:

$$
\eta_{e}=\frac{\tau_{11}-\tau_{22}}{\dot{\varepsilon}}
$$

In order to obtain the true elongational viscosity, the viscoelastic liquid must be free of any strain-history and the viscosity must be measured in a homogeneous flow field of constant extension rate. Fiber-spinning, of course, does not create such a flow field, as demonstrated by several investigators [14].

Analysis of any fluid flow must rely upon a constitutive equation that describes its rheological behavior accurately. A viscoelastic liquid, having finite (fading) memory, can well be approximated by an integral constitutive equation, which expresses the stress tensor explicitly in terms of local and upstream deformations and kinematics along the liquid particle's streamline. One such equation is the nonlinear integral constitutive equation proposed by Papanastasiou et al. [18] (the PSM model):

$$
\begin{aligned}
\tau(t)= & \int_{-\infty}^{t} \sum_{k} \frac{a_{k}}{\lambda_{k}} \exp \left(-\frac{t-t^{\prime}}{\lambda_{k}}\right) \\
& \times \frac{\alpha}{(\alpha-3)+\beta I+(1-\beta) I I} C_{t}^{-1}\left(t^{\prime}\right) d t^{\prime},
\end{aligned}
$$

where $\tau$ is the stress tensor at time $t, \lambda_{k}$ and $a_{k}$ the relaxation times and coefficients of the viscoelastic liquid, $C_{t}^{-1}\left(t^{\prime}\right)$ the Finger tensor, and $\alpha$ and $\beta$ material constants ( $\alpha$ is a shear parameter determined from stress-relaxation experiments at a limited number of large shear-strain levels and $\beta$ is an elongational parameter deduced from normal stress-relaxation tests at a limited number of large compressional or elongational strains). This constitutive equation successfully accounts for response in transient and steady shear flow, biaxial extensional flow, and uniaxial extensional flow [18]; therefore the equation has the potential for calculating the true elongational viscosity under well-defined experimental conditions, i.e., constant extension rate of uniaxial elongational flow starting from a virgin, unstressed state. The same equation accurately predicts the velocity (and fiber) profile and the drawing force in the spinning of polystyrene and polyethylene melts [17]; therefore, the equation duplicates the experimentally measured, apparent elongational viscosity of fiber-spinning.

The purpose of this study is to first highlight and then roughly quantify the difference between the true elongational viscosity and the one measured by fiberspinning-type rheometers, both as predicted by Eq. (2). 


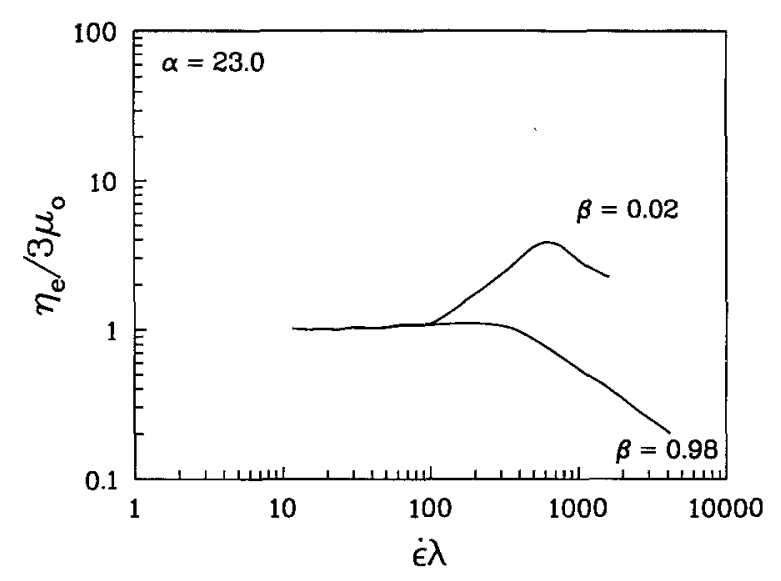

Fig. 1. The apparent elongational viscosity of a polymer of dimensionless relaxation time, $\lambda V / L=2.67$, and shearthinning parameter, $\alpha=23$, predicted by the PSM model in fiber-spinning of draw velocity $V$ and fiber length $L$

\section{Fiber spinning and apparent elongational viscosity}

In order to obtain the apparent elongational viscosity by fiber spinning, first the fiber-spinning equations are solved by means of the constitutive equation and the velocity and stress profiles along the spinline are computed. Then, the apparent elongational viscosity along the fiber is calculated by

$$
\eta_{a}\left(\dot{\varepsilon}=\frac{d u}{d z}\right)=\frac{\tau_{11}(z)-\tau_{22}(z)}{d u(z) / d z}
$$

where $u$ is the axial velocity and $\tau_{11}$ and $\tau_{22}$ the extra stresses in the axial and the radial directions, respectively.
The details of the analysis can be found elsewhere $[17,20]$. Results and comparisons to experimental data are also contained in these papers; they indicate that the analysis accurately predicts the behavior of a specified a priori viscoelastic liquid (i.e., of fixed-material parameters estimated by standard rheological tests that do not include fiber-spinning) in fiber-spinning. Table 1 shows the measured and predicted results for two different materials. Velocity and fiber profiles are also predicted equally well, as shown in $[17,20]$.

From these comparisons it can be seen that modeling of fiber spinning with the PSM model is reasonably accurate. Therefore, the predicted apparent elongational viscosity, deduced from this data and the velocity profiles are expected to be accurate and therefore identical to the one calculated experimentally as the ratio of the normal-stress difference (given by the measured tension) to the local extension rate (calculated by the measured-velocity profile).

Predicted, apparent, elongational viscosities in fiber-spinning by the PMS model are of the type shown in Fig. 1 for an extension-thinning ( $b=0.98)$ and an extension-thickening $(b=0.02)$ polymer under elongational prehistory $[17,20]$. This family of curves is qualitatively similar to the ones predicted by the same constitutive equation under well-defined conditions (Fig. 12 of [18]).

\section{Comparison of true and apparent elongational viscosities}

Comparisons of the apparent elongational viscosity by fiber-spinning (e.g., Fig. 1) under elongational pre-

Table 1. Comparisons between experimental data and predictions of the PSM model

\begin{tabular}{lllll}
\hline Fiber-spinning & \multicolumn{2}{l}{ Polystyrene $\left(T=170^{\circ} \mathrm{C}\right)$} & & \multicolumn{2}{l}{ LDPE $\left(T=150^{\circ} \mathrm{C}\right)$} \\
\cline { 2 - 5 } & $\lambda_{k}(\mathrm{~s})$ & $a_{k}(\mathrm{~Pa})$ & $\lambda_{k}(\mathrm{~s})$ & $a_{k}(\mathrm{~Pa})$ \\
\hline & $1.77 \times 10^{2}$ & $9.78 \times 10^{1}$ & $3.62 \times 10^{2}$ & $1.59 \times 10^{1}$ \\
& $2.09 \times 10^{1}$ & $3.46 \times 10^{3}$ & $4.96 \times 10^{1}$ & $4.33 \times 10^{2}$ \\
& $3.93 \times 10^{0}$ & $6.78 \times 10^{3}$ & $1.21 \times 10^{1}$ & $2.76 \times 10^{2}$ \\
& $4.39 \times 10^{-1}$ & $1.01 \times 10^{4}$ & $5.17 \times 10^{0}$ & $7.61 \times 10^{3}$ \\
\hline Draw ratio & $5.05 \times 10^{-2}$ & $1.26 \times 10^{4}$ & $4.94 \times 10^{-1}$ & $2.45 \times 10^{4}$ \\
\hline & $4.55 \times 10^{-3}$ & $1.45 \times 10^{4}$ & $5.48 \times 10^{-2}$ & $2.29 \times 10^{4}$ \\
\hline Drawing force (N) & $5.85 \times 10^{-4}$ & $1.47 \times 10^{4}$ & 2.61 & 2.61 \\
\hline
\end{tabular}


history with the true elongational viscosity, calculated by means of Eq. (1), are shown in Fig. 2. The resulting apparent viscosity is close to the true one only at low Weissenberg numbers, roughly

$$
\text { We }<10 \text {. }
$$

The Weissenberg number for the multi-relaxation mode is defined by

$$
W e=\dot{\varepsilon} \lambda=\dot{\varepsilon} \frac{\sum a_{k} \lambda_{k}^{2}}{\sum a_{k} \lambda_{k}},
$$

where $\lambda_{k}$ and $a_{k}$ are relaxation times and coefficients and $\dot{\varepsilon}$ the local extension rate.

Two more comparisons are made between the apparent elongational viscosity and the true one for two real polymeric materials, polystyrene and low-density polyethylene, as predicted by the constitutive equation. The results are shown in Fig. 3, as predicted by the PSM model. It is obvious (by smooth extrapolation) that the apparent elongational viscosity measured by fiber-spinning is the true elongational viscosity only at low Weissenberg numbers, $W e<1$. It appears that the upper fiber-end, the rate of deformation is low, all materials exhibit Newtonian behavior independent of any history, and therefore the two viscosities agree. At the take-up end, the extension rate is high and the true elongational viscosity is underestimated for extension-thickening liquids by an order of magnitude. It is worthwhile noticing here

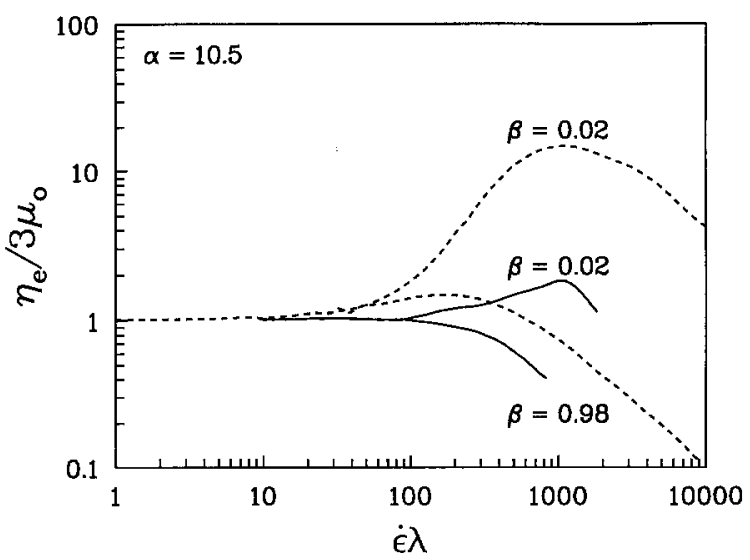

Fig. 2. Comparison between true elongational viscosity $(---)$ and apparent elongational viscosity (predicted by the PSM model at dimensionless relaxation time, $\lambda V / L=2.67$, and shear-thinning parameter $\alpha=10.5$

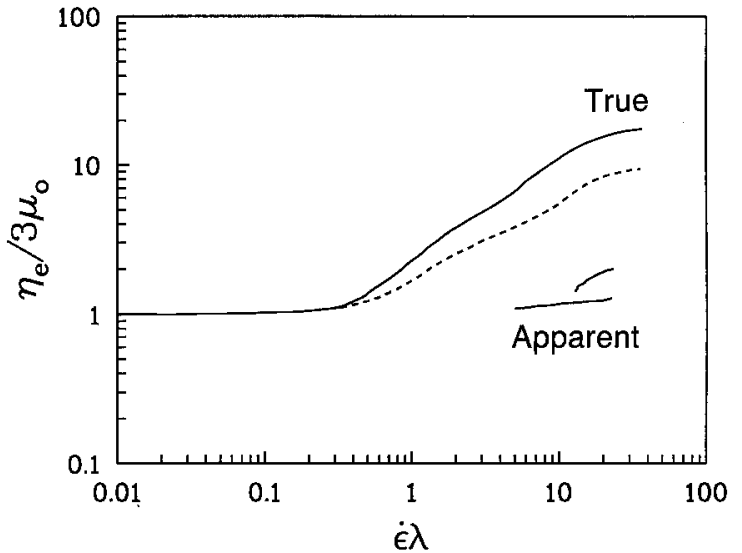

Fig. 3. Comparison between true elongational viscosity (PS ------, LDPE - - -) and apparent elongational viscosity (PS - - - , LDPE —) predicted by the PSM model for polystyrene and low density polyethylene [17]

that the qualitative behavior of the elongational viscosity is similar to that measured after imposition of a constant-extension rate to a material at rest (stress growth in start-up). In many ways, the two situations are similar if the length along the spinline is momentarily replaced by time. Unfortunately, there is no complete data in the literature for comparison which must include shear data to fit the material parameters of the constitutive equation, well-defined elongational data to estimate the true elongational viscosity, and fiber-spinning data to reproduce the apparent elongational viscosity for the same material at the same temperature. This is, of course, in addition

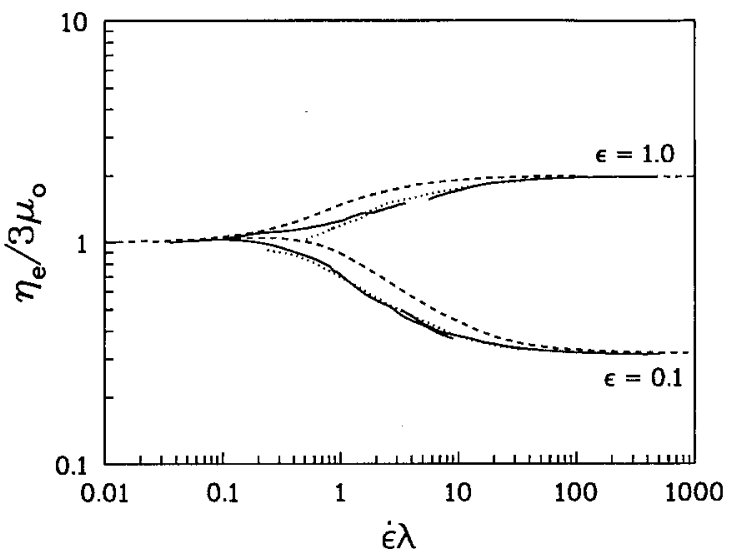

Fig. 4. Comparison between true elongational viscosity $\left(--_{-}\right)$and apparent elongational viscosity predicted by the Curtiss-Bird model for link tension coefficients $\varepsilon=0.1$ and $\varepsilon=1.0$ at $\lambda^{*}=0.01(-\cdot-), \lambda^{*}=0.1(\cdots \cdots)$, and $\lambda^{*}=1.0(\longrightarrow)$ in fiber-spinning, where $\lambda^{*}$ is a dimensionless relaxation time defined as $\lambda^{*}=\lambda V / L$ with $L$ the fiber length and $V$ the draw velocity 
to the uncertainty of existing techniques measuring true elongational viscosity.

Figure 4 shows corresponding curves predicted by the Curtiss-Bird [21] constitutive equation. The asymptotic predictions of the Curtiss-Bird model at large Weissenberg numbers are consistent with its predictions [27] in start-up of elongation, where it predicts elongational viscosities that are twice their zero-extension rate viscosity at most. Thus, this limitation of the model simply propagates in the fiberspinning viscosity, whereas the PSM model, which allows the elongational viscosity to grow up to ten times its steady shear viscosity, does not exhibit this behavior; instead the difference between true and apparent elongational viscosities continues to grow with the Weissenberg number.

\section{Conclusions}

The commonly known fact, that fiber-spinningtype rheometers do not necessarily measure true elongational viscosity, has been substantiated and roughly quantified by means of two accurate constitutive equations, which, in addition to spectrum of relaxation times, shear-thinning, and extension-thinning or thickening, can also account for the history of the deformation within the spinneret. The latter, together with the nonhomogeneous field of extension rate, experienced by the fluid particles along the spinline, give rise to an apparent, fiber-spinning, elengational viscosity, which may be off the true one at high elasticity by more than an order of magnitude.

These findings need validation by experiments, under shear and well-defined elongational tests, with the same material and at the same temperature. However, currently lacking such reliable data, the performance and the ability of the constitutive equations used to fit or predict extensive experimental data in many kinds of well-defined deformations provides a strong indication about the validity of these conclusions.

\section{References}

1. Spearot JA, Metzner AB (1972) Trans Soc Rheol 16:495

2. Zeichner GR (1973) Thesis, University of Delaware, Newark
3. Hudson NE, Ferguson J, Machie P (1974) Trans Soc Rheol 18:541

4. Mewis J, Gleyn GD (1982) AIChE J 28:900

5. Khagram M, Gupta RK, Sridhar T (1985) J Rheology 29:191

6. Sridhar T, Gupta RK (1985) Rheol Acta 24:207

7. Secor RB, Schunk PR, Hunter TB, Stitt SF, Macosko CW, Scriven LE (1989) J Rheology 33:1329

8. Pearson G, Middleman S (1977) AIChE J 23:714, 722

9. Winter HH, Macosko CW, Bennett KE (1979) Rheol Acta 18:323

10. Fuller GG, Leal LG (1980) Rheol Acta 19:580

11. Chatrai S, Macosko CW, Winter HH (1981) J Rheol $34: 433$

12. Mikkelsen KJ, Macosko CW, Fuller GG (1988) Proc Int Congr Rheol, Sydney

13. Chen I-J, Hagler GE, Abbott LE, Bogue DC, White JL (1972) Trans Soc Rheol 16:473

14. Petrie CJS (1978) J Non-Newt Fluid Mech 4:137

15. Larson RC (1983) J Rheol $27: 475$

16. Sridhar T, Gupta RD (1988) J Non-Newt Fluid Mech 27:349

17. Papanastasiou TC, Macosko CW, Scriven LE, Chen Z (1987) AIChE J 33:834

18. Papanastasiou TC, Scriven LE, Macosko CW (1983) J Rheol 27:387

19. Phan-Thien N (1978) J Rheol 22:259

20. Chen Z, Papanastasiou TC (1987) Intern Polym Proc $2: 33$

21. Curtiss CF, Bird RB (1981) J Chem Phys 74:2026

22. Doi M, Edwards SF (1979) J Chem Soc Faraday Trans 74:918

23. Ishizuka O, Koyama K (1980) 21:164

24. Meissner J, Raeble T, Stephenson SE (1981) J Rheol 25:1

25. Papanastasiou TC, Macosko CW, Scriven LE (1986) Int J Num Mech Fluids 6:819

26. MacKay ME, Petrie CJS (1989) Rheol Acta 28:281

27. Bird RB, Saab HH, Curtiss CF (1982) J Phys Chem 86:1102

(Received February 5, 1990; in revised form June 9, 1990)

Correspondence to:

Professor T. C. Papanastasiou

Department of Chemical Engineering

University of Michigan

Dow Building

Ann Arbor, MI 48109-2136

USA 